\title{
Low molecular weight heparin (nadroparin) improves placental permeability in rats with gestational diabetes mellitus via reduction of tight junction factors
}

\author{
YUEHUA SHI $^{1}$, JIE QIAN $^{2}$, FENG ZHANG $^{2}$, BEIBEI JIA ${ }^{3}$, XIAOYAN LIU ${ }^{4}$, YAN HU $^{1}$, \\ QINFEN ZHANG ${ }^{1}$, YE YANG ${ }^{5}$, DONGDONG SUN ${ }^{5,6}$ and LI JIANG ${ }^{7}$
}

\begin{abstract}
${ }^{1}$ Department of Obstetrics and Gynecology, Zhongda Hospital, School of Medicine, Southeast University, Nanjing, Jiangsu 210009; ${ }^{2}$ College of Pharmacy, Nanjing University of Chinese Medicine, Nanjing, Jiangsu 210023;

${ }^{3}$ Department of Pediatrics, The Affiliated Wuxi Maternity and Child Health Care Hospital of Nanjing Medical University,

Wuxi, Jiangsu 214002; ${ }^{4}$ Maternal and Child Health Care Center, Nanjing, Jiangsu 211100;

${ }^{5}$ Jiangsu Collaborative Innovation Center of Traditional Chinese Medicine Prevention and Treatment of Tumors;

${ }^{6}$ Translational Medicine Research Center, Nanjing University of Chinese Medicine, Nanjing, Jiangsu 210023;

${ }^{7}$ Department of Pediatrics, Zhongda Hospital, School of Medicine, Southeast University, Nanjing, Jiangsu 210009, P.R. China
\end{abstract}

Received March 6, 2019; Accepted October 15, 2019

DOI: $10.3892 / \mathrm{mmr} .2019 .10868$

\begin{abstract}
Placental structural abnormalities and dysfunction in those with gestational diabetes mellitus (GDM) can lead to increased placental permeability, which is in turn related to a poorer maternal and fetal prognosis. The present study sought to assess whether increased placental permeability in rats with GDM was accompanied by alterations in tight junction (TJ) factors and to evaluate the impact of low molecular weight heparin (LMWH) on these factors. The present study was conducted using pregnant female rats that were randomized into control, GDM and GDM + LMWH groups. Diabetes was induced via intraperitoneal administration of streptozotocin to rats in the GDM and GDM + LMWH groups, whereas rats in the GDM + LMWH group received daily subcutaneous LMWH starting on day 5 of pregnancy. On gestational day 16 , all rats were sacrificed and Evans Blue (EB) assay was used
\end{abstract}

Correspondence to: $\mathrm{Dr} \mathrm{Li}$ Jiang, Department of Pediatrics, Zhongda Hospital, School of Medicine, Southeast University, 87 Dingjia Qiao, Gulou, Nanjing, Jiangsu 210009, P.R. China E-mail: lijiang_su@163.com

Abbreviations: AGEs, advanced glycation end products; EB, Evans blue; GDM, gestational diabetes mellitus; IHC, immunohistochemistry; LMWH, low molecular weight heparin; OCLN, occludin; RAGE, receptor for advanced glycation end products; RT-qPCR, reverse transcription-quantitative PCR; TEM, transmission electron microscopy; TJ, tight junction; VEGF, vascular endothelial growth factor; VSM, vasculo-syncytial membrane; ZO-1, zonular occludens-1

Key words: GDM, placental permeability, TJ, RAGE, low molecular weight heparin, $\mathrm{NF}-\kappa \mathrm{B}$ to gauge vascular permeability based on EB dye leakage. Transmission electron microscopy was further used to assess TJ structures, and the TJ proteins zonular occludens- 1 (ZO-1) and occludin (OCLN) were assessed using immunohistochemistry and western blotting. Blood samples were obtained from the abdominal aorta for ELISA measurements of advanced glycation end products (AGEs) concentrations, and placental receptor for AGEs (RAGE) and vascular endothelial growth factor (VEGF) expression was assessed using reverse transcription-quantitative PCR. In addition, western blotting was used to measure placental NF- $\mathrm{kB}$. Compared with in the control group, EB leakage was markedly increased in GDM group rats; this was associated with reduced ZO-1 and OCLN expression. Conversely, LMWH attenuated this increase in placental permeability in rats with GDM and also mediated a partial recovery of ZO-1 and OCLN expression. Blood glucose and serum AGEs concentrations did not differ between the GDM and GDM + LMWH groups. Furthermore, LMWH treatment resulted in decreases in RAGE and VEGF mRNA expression levels, which were upregulated in the GDM group, whereas it had the opposite effect on the expression of NF- $\mathrm{kB}$. In conclusion, GDM was associated with increased placental permeability and this may be linked with changes in TJs. LMWH intervention mediated protection against this GDM-associated shift in placental permeability via the RAGE/NF- $\kappa$ B pathway.

\section{Introduction}

Gestational diabetes mellitus (GDM) is a form of diabetes that first manifests during pregnancy (1). Abnormalities of the placental barrier, including structural and functional dysfunction, are evident in the placenta of most women suffering from GDM in the form of abnormal glucose metabolism, and these changes can lead to adverse pregnancy outcomes $(2,3)$. The 
main structure of the placental barrier is the vasculo-syncytial membrane (VSM). The transcellular and paracellular pathways are the two main mechanisms whereby solutes are able to traverse the VSM. The syncytiotrophoblasts present on the maternal surface of the VSM lack any obvious fluid-filled paracellular space, but there is an inverse relationship between the rate of diffusion of inert hydrophilic solutes on the placenta and their molecular size, which suggests that a paracellular route exists at the VSM (4). Paracellular permeability is regulated by tight junctions (TJs) and adherens junctions, and by the proteins that make up these adhesive cell-cell junction points. TJs have been reported to be present in both trophoblast cells and in fetal vessel epithelium (5).

Previous studies have suggested that placental dysfunction in patients with GDM is caused by hyperglycemia $(6,7)$. In addition, other studies have identified that insulin therapy does not improve fetal or newborn metabolic outcomes (8), and that it can cause alterations in the placenta (9), indicating factors other than glucose may be involved in the pathophysiology of GDM. In a number of studies, advanced glycation end products (AGEs) have been identified to be present in higher levels in women suffering from $\operatorname{GDM}(10,11)$ and these products are associated with poor fetal outcomes (12). AGEs are reportedly involved in increasing the permeability of retinal vascular and endothelial cells $(13,14)$. AGEs exert their deleterious effects by either directly damaging cells or by binding to the specific receptor for AGEs (RAGE). The binding of AGEs with RAGE stimulates NF- $\kappa B$ pathway activation (15), enhancing the release of vascular endothelial growth factor (VEGF) (16).

It was therefore hypothesized that AGEs may be an important factor worthy of study in the context of placental barrier dysfunction in patients with GDM. The present study evaluated placental permeability and the expression of TJ-associated proteins in rats with GDM, and investigated the association of GDM with alterations in AGEs, RAGE, NF- $\mathrm{KB}$ and VEGF. In addition, low molecular weight heparin (LMWH), as an anticoagulant capable of reducing the risk of recurrent placenta-mediated pregnancy complications (17), has a potent effect on the vascular endothelium $(18,19)$. Kevane et al $(20)$ identified that the LMWH tinzaparin serves a protective role in endothelial barrier function. As such, LMWH was employed to assess whether it had the ability to reduce placental permeability and to evaluate its relationship with RAGE.

\section{Materials and methods}

Materials. A total of 35 healthy 12 -week-old female Sprague-Dawley (SD) rats (250-300 g) and 15 adult male SD rats (300-350 g) were acquired from Beijing Vital River Laboratory Animal Technology Co., Ltd. Animals were maintained in a controlled environment $\left(25 \pm 1^{\circ} \mathrm{C}, 50 \%\right.$ humidity and 12-h light/dark cycle) and were given free access to water and a standard laboratory diet. The Medical Ethics Committee of Southeast University approved all animal studies.

The primary antibodies used in this study were: Rabbit anti-zonular occludens-1 (ZO-1) (cat. no. 61-7300; Invitrogen; Thermo Fisher Scientific, Inc.), rabbit anti-occludin (OCLN; cat. no. ab216327; Abcam), rabbit anti-NF-кB p65 (cat.no. ab16502; Abcam), rabbit anti-GAPDH (cat.no. ab9485; Abcam) and horseradish peroxidase (HRP)-conjugated goat anti-rabbit secondary antibody (cat. no. ab130805; Abcam). The LMWH used was nadroparin calcium injection, which was purchased from GlaxoSmithKline plc. Bull serum albumin (BSA) was purchased from Gibco (Thermo Fisher Scientific, Inc.).

Animal model preparation. Female rats in estrus were allowed to cohabitate for one night with male rats at a 2:1 ratio in order to facilitate conception; female rats with detectable sperm in their vaginal smear were considered pregnant (day 0). The 30 pregnant rats were randomized into three groups ( $n=10 /$ group): Normal control (NC) group, GDM group and GDM + LMWH group. GDM was induced in animals via intraperitoneally injecting a single dose of streptozotocin $(45 \mathrm{mg} / \mathrm{kg}$; Wako Pure Chemical Industries, Ltd.), which had been freshly prepared. Blood glucose levels of all rats were detected with a glucometer (Onetouch, Ultra, Johnson \& Johnson). Rats exhibiting a glucose concentration of $>16.7 \mathrm{mmol} / 1$ for 3 days after the injection were used in this study. NC animals were instead injected with $1 \mathrm{ml}$ sodium citrate buffer. On gestational day 5, rats in the GDM + LMWH group were injected subcutaneously with LMWH $(600 \mathrm{IU} / \mathrm{kg} / \mathrm{d})$ to establish an LMWH intervention model, while other groups were administered an equal volume of $0.9 \%$ saline. On day 16 of pregnancy, animals were sacrificed and the placentas were removed for western blotting, reverse transcription-quantitative (RT-q)PCR, transmission electron microscopy (TEM) and immunohistochemistry (IHC).

ELISA. Blood samples $(1 \mathrm{ml})$ were obtained from the abdominal aorta on day 16 after sacrifice and were centrifuged at 2,000 x g for $20 \mathrm{~min}$ at $5^{\circ} \mathrm{C}$. Concentrations of AGEs were measured from the collected samples using a rat AGEs ELISA kit (cat. no. RA20685; Bioswamp Wuhan Beinle Biotechnology Co., Ltd.), according to the manufacturer's protocol. The sensitivity of this assay was $<6 \mathrm{ng} / \mathrm{ml}$, whereas the coefficient of intraplate variation was $\leq 9 \%$, with interassay variation $\leq 11 \%$. AGEs were detected using a microplate reader (ELx800; BioTek Instruments, Inc.) at an emission wavelength of $450 \mathrm{~nm}$. Standard curves were used to calculate AGEs concentrations $(\mathrm{ng} / \mathrm{ml})$ in serum.

Evans Blue (EB) assay. An EB assay was used to measure VSM leakage. EB (50 mg/kg; Sigma-Aldrich; Merck KGaA) was injected into the tail vein in $2 \%$ PBS. Rats were sacrificed 45 min after injection of EB and the placentas were harvested, weighed, and incubated in $1 \mathrm{ml}$ formamide at $55^{\circ} \mathrm{C}$ for $24 \mathrm{~h}$ to extract EB from tissues. The supernatant was collected after centrifugation at $12,000 \mathrm{xg}$ for $0.5 \mathrm{~h}$ at $4{ }^{\circ} \mathrm{C}$ and a microplate reader was used to measure absorbance at $630 \mathrm{~nm}$. EB content in placental samples (in $\mathrm{ng} / \mathrm{ml}$ ) was calculated based upon a standard curve using the following formula: Placenta EB content $(\mathrm{ng} / \mathrm{mg})=$ sample EB content $(\mathrm{ng} / \mathrm{ml}) \times$ formamide volume (ml)/placental weight (mg) (21).

$T E M$. The ultrastructure of placentas was examined by TEM. The placental samples were placed in cacodylate buffer with $0.05 \mathrm{M}$ sucrose and stored at $4^{\circ} \mathrm{C}$ for $24 \mathrm{~h}$, following fixation with $2.5 \%$ glutaraldehyde in cacodylate buffer at $4^{\circ} \mathrm{C}$ for $1 \mathrm{~h}$. The placental samples were fixed again in $1 \%$ osmium 
A
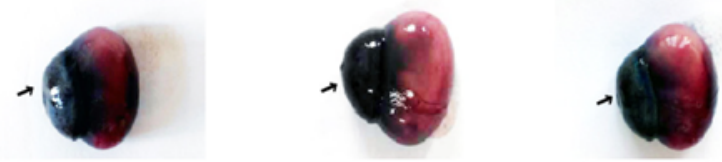

B

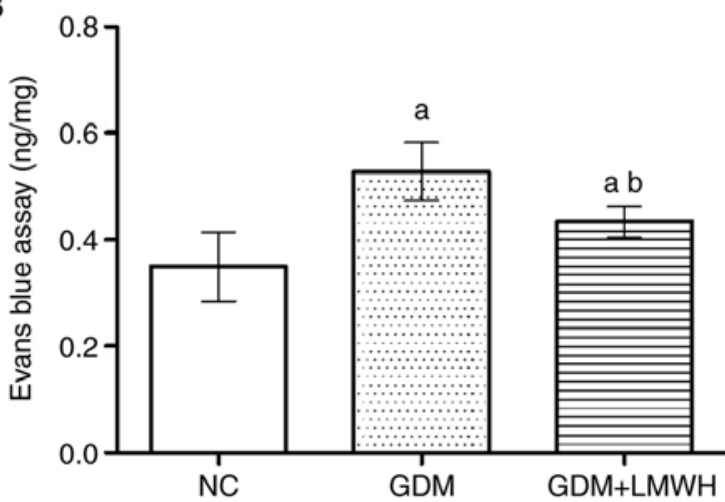

Figure 1. EB content in the placentas in each group of rats. (A) Placentas were removed from rats by cesarean section after Evans Blue assay on day 16 of pregnancy in each group. Black arrows point to the rat placentas. (B) Content of EB in the placenta of each group on day 16 of pregnancy. Data are presented as the means \pm standard deviation. ${ }^{a} \mathrm{P}<0.05$ vs. NC group, ${ }^{\mathrm{b}} \mathrm{P}<0.05$ vs. GDM group. EB, Evans blue; GDM, gestational diabetes mellitus; LMWH, low molecular weight heparin; NC, normal control.

tetroxide for $1 \mathrm{~h}$ at $4^{\circ} \mathrm{C}$ after dehydration treatment with a graded ethanol series, and then placed into in a graded series of acetone. Samples were then sliced into sections $(1 \mu \mathrm{m})$ after embedding in araldite. The sections were dyed with $2 \%$ uranyl acetate for $10 \mathrm{~min}$ and then with $100 \mu \mathrm{l}$ lead citrate for $5 \mathrm{~min}$ at $25^{\circ} \mathrm{C}$. The placental TJ ultrastructure was systematically investigated via DigitalMicrograph 3.9 (Gatan Inc.) by electron microscope (EVO MA 10; Zeiss GmbH) at x25,000 magnification.

$I H C$. IHC was conducted to investigate differences in the expression of ZO-1 and OCLN in placental tissue. Placental samples were fixed in formalin at $22^{\circ} \mathrm{C}$ for $6 \mathrm{~h}$, dehydrated by different concentration gradients of ethanol, be transparent in xylene for $30 \mathrm{~min}$, paraffin-embedded, and sectioned at $5 \mu \mathrm{m}$. The paraffin slices were dewaxed and rehydrated by xylene and with a graded ethanol series, then treated with $3 \% \mathrm{H}_{2} \mathrm{O}_{2}$ for $10 \mathrm{~min}$, followed by incubation with appropriate primary antibodies (dilution: Rabbit anti-ZO-1, 1:200; rabbit anti-OCLN, 1:500) for $24 \mathrm{~h}$ at $4^{\circ} \mathrm{C}$. Secondary antibodies (1:500) were then used to probe samples for $50 \mathrm{~min}$ at $37^{\circ} \mathrm{C}$. The sections were then restained with $1 \%$ hematoxylin at $22^{\circ} \mathrm{C}$ for 1 min, dehydrated, cleared and mounted. An automated light microscope (DMLA, Leica Microsystems $\mathrm{GmbH}$ ) was used to detect the location of dyeing area.

Western blotting. RIPA buffer [5 mM EDTA, $150 \mathrm{mM} \mathrm{NaCl}$, $3 \mu \mathrm{PMSF}, 1 \% \mathrm{NP} 40$ and $50 \mathrm{mM}$ Tris- $\mathrm{HCl}$ (pH 7.0)] was used for protein extraction. BCA protein assay kit (P0009, Beyotime Institute of Biotechnology) was used to quantify the protein of each samples. Equivalent protein amounts from each sample $(4.25 \mu \mathrm{g})$ were then separated via SDS-PAGE with $10 \%$ gels, followed by transfer onto a PVDF membrane (GE Healthcare Life Sciences). Blots were washed three times using TBS- $0.1 \%$
Tween (TBST; $10 \mathrm{~min} /$ wash), blocked using 5\% fat-free milk in TBST at $22^{\circ} \mathrm{C}$ for $4 \mathrm{~h}$ and then probed overnight at $4^{\circ} \mathrm{C}$ with primary antibodies (dilution: Rabbit anti-ZO-1, 1:1,000; rabbit anti-OCLN, 1:2,000; rabbit anti-NF- $\mathrm{B}$ p65 1:500; rabbit anti-GAPDH, 1:2,500). Blots were again washed three times with TBST and then probed with secondary HRP-conjugated goat anti-rabbit IgG (1:3,000 in TBST containing 10\% BSA) at $22^{\circ} \mathrm{C}$ for $1 \mathrm{~h}$. An enhanced chemiluminescence kit (Tanon 5200; Tanon Science and Technology Co., Ltd.) was used for protein visualization. Images were recorded and data were analyzed with Image J 1.8.0 (National Institutes of Health).

$R T$ - $q P C R$. RAGE and VEGF-A mRNA expression levels were assessed using total RNA that was extracted from placental homogenates using TRIzol ${ }^{\circledR}$ (Invitrogen; Thermo Fisher Scientific, Inc.) based on manufacturer's protocols. The first-strand complementary synthesis reaction was performed using a PrimeScript RT Reagent Kit (RR047A; Takara Biotechnology Co., Ltd.). RAGE and VEGF expression were detected using the SLAN real-time PCR detection system (Shanghai Hongshi Medical Technology Co., Ltd.). The primer sequences were as follows: VEGF, forward 5'-CAATGATGA AGCCCTGGAGTG-3', reverse 5'-GCTCATCTCTCCTAT GTGCTGG-3'; RAGE, forward 5'-TGAGACGGGACTCTT CACGCT-3', reverse 5'-CACCTTCAGGCTCAACCAACA-3'; and $\beta$-actin, forward 5'-TGCTATGTTGCCCTAGACTTCG-3' and reverse 5'-GTTGGCATAGAGGTCTTTACGG-3'. $\beta$-actin was used as an internal control. Amplification conditions were: $95^{\circ} \mathrm{C}$ for $10 \mathrm{~min}$ for enzyme activation, 40 cycles of denaturation at $95^{\circ} \mathrm{C}$ for $15 \mathrm{sec}$, annealing at $60^{\circ} \mathrm{C}$ for $10 \mathrm{~min}$, and dissociation curve assessment between $75^{\circ} \mathrm{C}$ and $95^{\circ} \mathrm{C}$ with continuous fluorescence measurement. Only RNA samples with an OD260/OD280 ratio of 1.8-2.0 were used for RT. Each sample was measured in triplicate and the $2^{-\Delta \Delta \mathrm{Cq}}$ method (22) was used to assess relative gene expression.

Statistical analysis. All data were presented as the means \pm standard deviation of triplicate experiments. SPSS 21.0 (SPSS, Inc.) was used for all analyses, with analysis of variance via one-way analysis of variance followed by post-hoc Student-Newman-Keuls test. $\mathrm{P}<0.05$ was considered to indicate a statistically significant difference.

\section{Results}

Effects on placental permeability as measured by EB assay. EB is a dye with a high affinity for albumin, which cannot pass the endothelium; therefore, EB bound to albumin is restricted to blood vessels. In some pathological conditions associated with high vascular permeability, endothelial cells partially lose their TJs and allow small molecules such as albumin to pass through (21), thereby allowing EB leakage into tissues. In the present study, EB was injected into the tail vein of rats to evaluate paracellular permeability of the placenta in each group.

Upon examination, it was evident that placental coloration differed between groups, with higher EB content corresponding to a darker color (Fig. 1A). Furthermore, optical density analysis revealed that EB leakage in GDM group placentas was $\sim 1.5$-fold higher than in the NC group $(\mathrm{P}<0.05)$. 
$\mathrm{C}$

A

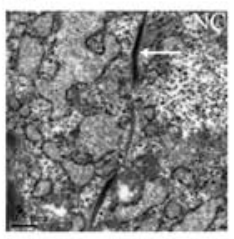

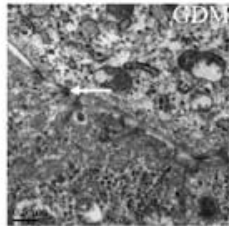
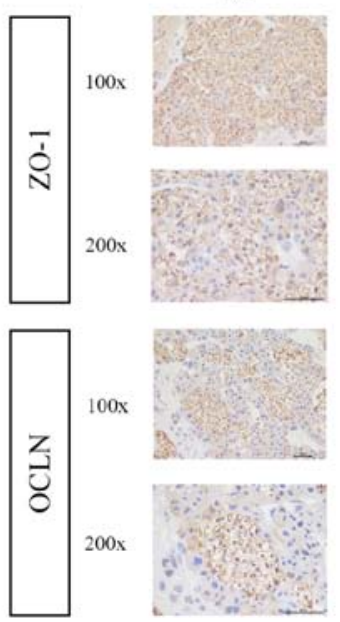

GDM
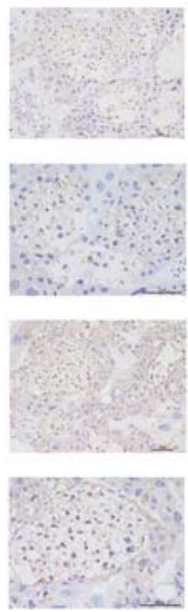

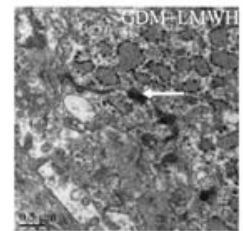

GDM-LMWH
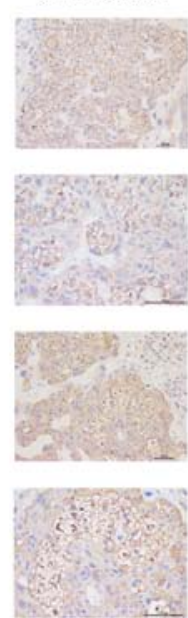

NC

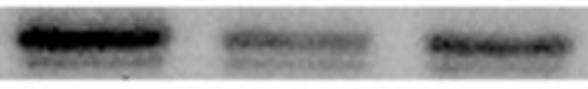

ZO-

Occludin

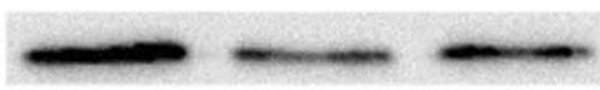

$N F-\kappa B$

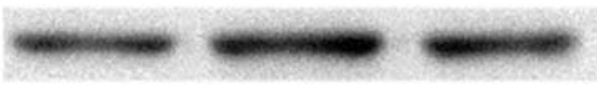

GAPDH

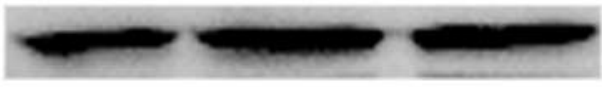

$\mathrm{D}$

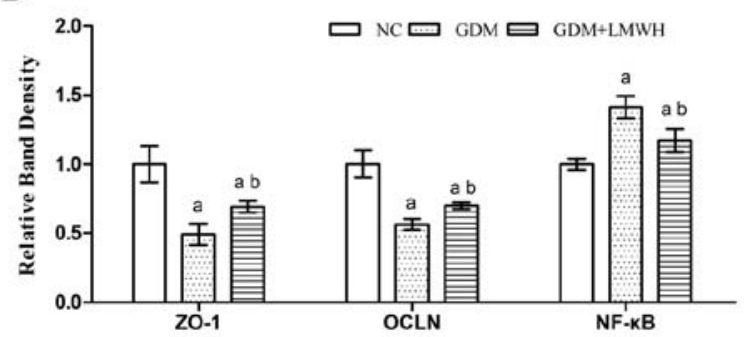

Figure 2. Alterations in the structure, location and expression of tight junctions in the placentas of each group. (A) Electron micrographs of the placental ultrastructure in each group were captured via transmission electron microscopy at x 25,000 magnification (scale bar, $0.5 \mu \mathrm{m}$ ). White arrows indicate the TJs on the membrane between cells. (B) Expression and localization of ZO-1 and OCLN in placental tissues were observed via IHC (scale bar, $100 \mu \mathrm{m}$ ). Images in the first row correspond to x100 magnification, while those in the second row correspond to x200 magnification. (C) Representative western blotting results

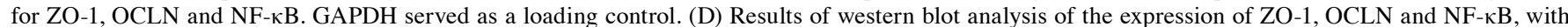
semi-quantification shown. Data represent fold changes relative to the NC group. Data are presented as the means \pm standard deviation from three independent experiments. ${ }^{\mathrm{a}} \mathrm{P}<0.05$ vs. NC group, ${ }^{\mathrm{b}} \mathrm{P}<0.05$ vs. GDM group. GDM, gestational diabetes mellitus; LMWH, low molecular weight heparin; $\mathrm{NC}$, normal control; OCLN, occludin; TJ, tight junction; ZO-1, zonular occludens-1.

LMWH-treated GDM rats exhibited lower EV leakage compared with in the GDM group $(\mathrm{P}<0.05)$, although this leakage was still higher compared with the $\mathrm{NC}$ group $(\mathrm{P}<0.05$; Fig. 1B).

Alteration of placental TJ structure. The ultrastructure of TJs was assessed by TEM. Under TEM, TJs between syncytiotrophoblasts and vascular endothelial cells in the placental tissue were loose and damaged in placental samples from the GDM group. In contrast, animals treated with LMWH exhibited reduced placental structural damage relative to animals in the GDM group (Fig. 2A).

TJ protein expression analysis. IHC was used to observe the localization and expression of TJ proteins, including $\mathrm{ZO}-1$ and OCLN. As expected, ZO-1 and OCLN were mostly located in the syncytiotrophoblast cell membrane, localizing to the TJs between cells. The two proteins did not exhibit apparent redistribution in the GDM group or the GDM + LMWH group compared with in the NC group (Fig. 2B). The expression levels of ZO-1 and OCLN were lower in the GDM group compared with in the $\mathrm{NC}$ group.

Western blotting was used to confirm differences in OCLN and ZO-1 protein levels, demonstrating clear reductions in these proteins in GDM placental tissues compared with in the NC group $(\mathrm{P}<0.05)$ (Fig. 2C and D), whereas LMWH upregu- lated the expression levels of these proteins $(\mathrm{P}<0.05)$, albeit to levels lower compared with the NC group $(\mathrm{P}<0.05)$.

Increased blood glucose and serum AGEs levels in the GDM model. On day 16 of pregnancy, blood glucose and serum AGEs levels were assessed in all groups. Animals in the GDM and GDM + LMWH groups exhibited significantly higher blood glucose levels compared with in the NC group $(\mathrm{P}<0.05)$, but no significant differences were observed between the GDM and GDM + LMWH groups ( $>>0.05$; Fig. 3A). AGEs serum levels exhibited variations similar to those of blood glucose in the GDM and GDM + LMWH groups (Fig. 3B).

Altered placental $R A G E / N F-\kappa B$ pathway activation. In order to clarify the signaling pathways associated with altered placental permeability in this model, the RAGE/NF- $\kappa \mathrm{B}$ signaling pathway was analyzed in the placental samples. The mRNA expression levels of RAGE and VEGF were assessed via RT-qPCR. RAGE expression was significantly increased in samples from the GDM and GDM + LMWH groups compared with in the $\mathrm{NC}$ group $(\mathrm{P}<0.05)$. LMWH treatment partially decreased this increase in RAGE expression in animals with GDM ( $\mathrm{P}<0.05$; Fig. 4A). Compared to normal rats, GDM rats exhibited a significant upregulation of VEGF mRNA $(\mathrm{P}<0.05)$, whereas LMWH treatment reduced VEGF expression to levels similar to in the NC group $(\mathrm{P}<0.05$; Fig. 4B). These results 
A

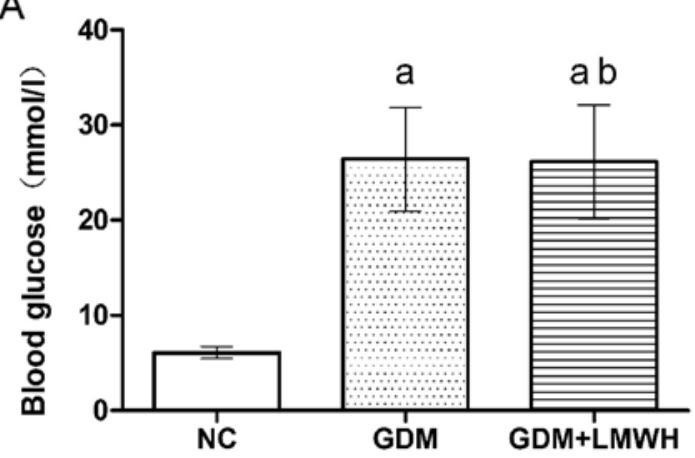

B

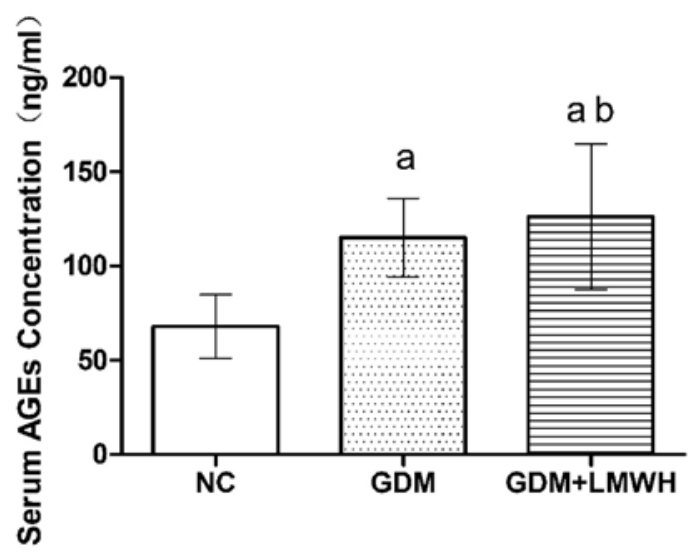

Figure 3. Levels of blood glucose and serum AGEs in each group of rats. (A) Blood glucose levels were tested with glucose oxidase. (B) Levels of serum AGEs tested by ELISA. Data are expressed as the means \pm standard deviation. ${ }^{\mathrm{a} P}<0.05$ vs. $\mathrm{NC}$ group, ${ }^{\mathrm{b}} \mathrm{P}>0.05$ vs. GDM group. AGEs, advanced glycation end products; GDM, gestational diabetes mellitus; LMWH, low molecular weight heparin; NC, normal control.

suggested that LMWH treatment may reduce the expression of RAGE and VEGF at the mRNA level.

$\mathrm{NF}-\kappa \mathrm{B}$ protein levels were assessed via western blotting (Fig. 2C). Compared with in NC animals, rats in the GDM group exhibited significantly increased placental NF- $\kappa$ B levels $(\mathrm{P}<0.05)$. NF- $\kappa \mathrm{B}$ levels were reduced following treatment with LMWH in GDM rats $(\mathrm{P}<0.05)$ to levels higher compared with $\mathrm{NC}$ animals $(\mathrm{P}<0.05$; Fig. 2D).

\section{Discussion}

The present study established a rat model of GDM to observe the changes in placental permeability and TJs, the effects of LMWH on these parameters, and the potential underlying mechanisms. It was identified that the GDM rats exhibited a significant increase in EB leakage and a lower expression of the TJ proteins OCLN and ZO-1 compared with in NC animals. Significant changes in the placentas of patients with GDM have previously been identified, including decreased syncytiotrophoblasts apical microvilli density, a thickened placental barrier and increased ST vacuoles (23). Hayward et al (24) observed changes in placental glucose and neutral amino acids in the context of GDM; however, the changes in placental transfer of macromolecules, such as albumin, in GDM remain to be elucidated The leakage of EB bound to albumin in the
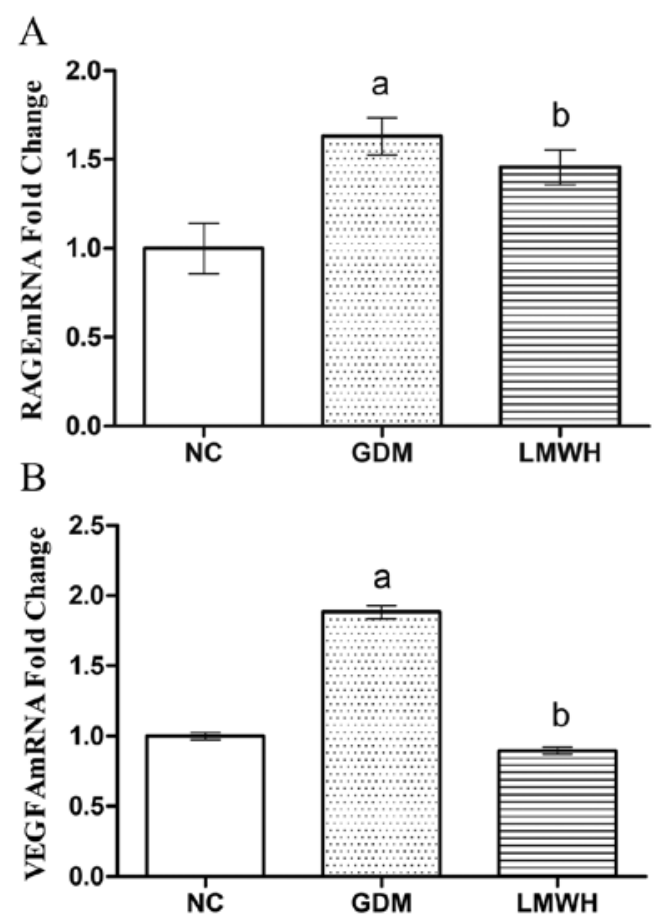

Figure 4. mRNA expression levels of RAGE and VEGF in placentas of each group. (A) Results of RT-qPCR analysis of the expression of RAGE mRNA. Data represent fold changes relative to the NC group. Data are presented as the means \pm standard deviation from three independent experiments. ${ }^{a} \mathrm{P}<0.05$ vs. NC group, ${ }^{b} \mathrm{P}<0.05$ vs. GDM group. (B) Results of RT-qPCR analysis of the expression of RAGE mRNA. ${ }^{\mathrm{a}} \mathrm{P}<0.05$ vs. NC group, ${ }^{\mathrm{b}} \mathrm{P}<0.05$ vs. GDM group. LMWH, low molecular weight heparin; RAGE, receptor for advanced glycation end products; RT-qPCR, reverse transcription-quantitative PCR; VEGF, vascular endothelial growth factor.

present study provided evidence of the transfer of albumin through the placenta. Reductions in TJ proteins in the placenta of GDM indicate dysfunction of the TJ barriers, thus increasing macromolecule flux via the paracellular route $(6,25)$. The results of TEM and IHC in the present study identified that TJs were located in endothelial and trophoblast cells, which is consistent with previous studies $(25,26)$. GDM placentas have been shown to exhibit a significant reduction in the TJ proteins, ZO-1 and OCLN, and the adherens junction proteins, VE-cadherin and $\beta$-catenin, particularly upon exposure to hyperglycemia during the first trimester when the vascular remodeling phase of placental growth occurs (25). Alterations in placental permeability and the expression of $\mathrm{TJ}$ proteins in GDM placentas may account for adverse fetal and neonatal outcomes $(6,8)$.

In the current study, the blood glucose and serum AGEs levels in GDM rats were higher compared with in normal rats. Previous work by Li and Yang (27) indicated that the level of serum AGEs was positively associated with glucose levels. Chronic hyperglycemia has been highlighted as a possible contributing factor in diabetic vascular complications (28) and may be the cause of increased vascular permeability in the context of diabetic retinopathy (29). Under conditions of chronic hyperglycemia, AGEs are actively formed and accumulate in circulation and in various tissues, as they are produced due to the non-enzymatic glycation of proteins, lipids and nucleic acids (30). The diabetic vascular complica- 
tions triggered by AGEs are dominant and independent of hyperglycemia in those with diabetes (31). Previously, AGEs were reported to increase the permeability of retinal vascular and endothelial cells by upregulating certain chemokines or promoting actin rearrangement (32). In Alzheimer's disease, AGEs have been identified as disrupting TJs in the blood-brain barrier, thus increasing its permeability (33). Therefore, both hyperglycemia and AGEs have an effect on vascular permeability.

AGEs exert their deleterious effects by either directly damaging cells or through a receptor-mediated pathway (15). RAGE is the most studied receptor of AGEs and is a member of the immunoglobulin superfamily, present primarily on vascular, endothelial and smooth muscle cells, and on monocyte/macrophage membranes (34). Once AGEs are recognized by RAGE on the cell membrane, downstream signaling leads to oxidative stress and inflammation in cells via the activation of NF- $\kappa \mathrm{B}$ (35). The expression of RAGE can be upregulated by AGEs, as evidenced by the fact that RAGE protein levels are increased in settings where AGEs are abundant (15). The present study identified that the expression of placental RAGE mRNA was increased in GDM group animals that exhibited elevated levels of serum AGEs. NF- $\kappa \mathrm{B}$ is a downstream target of RAGE and was significantly elevated in the GDM group in the present study. Mice with enhanced NF- $\kappa \mathrm{B}$ expression exhibit greater sensitivity to lipopolysaccharide-induced toxemia, which is associated with an increase in vascular permeability and a clear reduction in the formation of TJs (35). Upon activation of RAGE, the transcription factor $N F-\kappa B$ undergoes nuclear translocation and binds to the promoter region of RAGE, thereby inducing RAGE gene expression (15). It was therefore inferred that the RAGE/NF- $\kappa \mathrm{B}$ pathway was activated in GDM placentas in response to high AGEs levels.

As a master regulator of inflammation, $N F-\kappa B$ is capable of controlling the transcription of a range of genes related to the inflammatory response, including VEGF, which is known to enhance vascular permeability (36). VEGF binding to its cell surface receptors triggers the disassembly of TJs and promotes an increase in vascular permeability (36). Increased vascular permeability, deficiency of VE-cadherin and elevated levels of VEGF have been reported in patients with GDM $(26,37)$. Previous studies of barrier systems, such as the blood-brain barrier (33), the blood-retinal barrier (13) and the blood-placenta barrier (26), have confirmed the relationship between VEGF and TJs. Research into the blood-brain barrier has revealed that the expression of VEGF is upregulated by hypoxia, thereby increasing blood-brain barrier permeability (33). In addition, blood-retinal barrier dysfunction has been recovered by targeting ZO-1 through a reduction in VEGF (38). Hypoxia-induced TJ dysfunction in trophoblasts can be improved by downregulation of VEGF (26). A recent study identified that $\mathrm{VEGF}_{165 \mathrm{~b}}$, intercellular adhesion molecule 1 (ICAM-1) and AGEs in GDM were higher, and $\mathrm{VEGF}_{165 \mathrm{~b}}$ /total VEGF ratio was higher in GDM, which was correlated with ICAM-1 and AGEs (39). The present study also identified that the expression of VEGF-A mRNA was increased in the placentas of GDM rats, but did not detect the expression of each isoform of VEGF-A. VEGF ${ }_{165}$ and $\mathrm{VEGF}_{121}$ are the most highly expressed VEGF-A isoforms, whereas $\mathrm{VEGF}_{121}$ has no affinity for heparin (40). Therefore it was hypothesized that $\mathrm{VEGF}_{165}$ serves a major role in regulating placental permeability in GDM. It was further hypothesized that the RAGE/NF- $\kappa \mathrm{B}$ pathway in the placenta of animals with GDM can upregulate VEGF expression, making this an important mechanism underlying dysfunction at the GDM placental barrier.

The present study reported that LMWH attenuated the increase in VSM permeability in rats with GDM; this was accompanied by a partial recovery in the expression of ZO-1 and OCLN. There was no significant difference in blood glucose or serum AGEs concentrations between GDM and GDM + LMWH animals, indicating that LMWH has no role in regulating blood glucose or serum AGEs. This effect may instead be due to the non-anticoagulant effects of LMWH (40). According to previous studies, nadroparin, which was used in the present study, has anti-inflammatory, anti-metastatic and anti-fibrotic activities (41-44). Yalniz et al (44) reported that nadroparin exerts anti-oxidative and anti-inflammatory effects by regulating the $\mathrm{NF}-\kappa \mathrm{B}$ and nuclear factor erythroid 2-related factor 2 /heme oxygenase 1 pathways. In contrast to tinzaparin, fondaparinux or to direct oral anticoagulants, enoxaparin increases the permeability of podocytes to albumin, as reported by Delézay et al (45). The heterogeneity of LMWHs may explain these inconsistent and even opposite conclusions.

In the present study, LMWH intervention resulted in a decrease in RAGE and VEGF mRNA expression levels, which were upregulated in animals in the GDM group, whereas it markedly reduced $\mathrm{NF}-\kappa \mathrm{B}$ protein levels. The LMWH tinzaparin has been identified to serve a protective role in endothelial barrier function (20). Bentzer et al (46) revealed that heparins are potential inhibitors of hypertension-induced increases in vascular permeability.

LMWH has been reported to be a competitive antagonist of RAGE that competes with AGEs for RAGE binding, inhibiting its activation. This was identified by Takeuchi et al (47), who demonstrated that LMWH attenuates changes in diabetic kidneys by inhibiting RAGE. This finding suggests that LMWH may serve as an inhibitor of RAGE, which would alter the placental permeability in GDM. It was hypothesized that LMWH acts as a competitive antagonist of RAGE, whose expression was downregulated, thereby inactivating the RAGE/NF- $\kappa$ B pathway. Furthermore the expression of VEGF was decreased, thereby reducing placental permeability. A limitation of the present study is the lack of an in vitro study to further confirm the relationship between GDM and LMWH. In addition, the effects of GDM on the offspring were not analyzed.

In conclusion, the AGEs-RAGE system may represent a novel target for treating placental barrier dysfunction in GDM. LMWH may be a potential drug for the treatment of diseases related to the AGEs-RAGE system.

\section{Acknowledgements}

Not applicable.

\section{Funding}

The present study was supported by The National Natural Science Foundation of China (grant no. 659000095) and 
Science and Technology Development Foundation of Nanjing Medical University (grant no. NMUB2018249).

\section{Availability of data and materials}

All data generated or analyzed during this study are included in this published article.

\section{Authors' contributions}

YS, JQ, FZ and BJ performed experiments and data analysis; YS, BJ and JQ drafted the manuscript; YS, QZ, XL, YH, YY, DS and LJ participated in the study design, data collection and revision process. All authors read and approved the final manuscript.

\section{Ethics approval and consent to participate}

The Medical Ethics Committee of Southeast University approved all animal studies.

\section{Patient consent for publication}

Not applicable.

\section{Competing interests}

The authors declare that they have no competing interests.

\section{References}

1. American Diabetes Association: Diagnosis and classification of diabetes mellitus. Diabetes Care 37 (Suppl 1): S81-S90, 2014

2. Jarmuzek P, Wielgos M and Bomba-Opon D: Placental pathologic changes in gestational diabetes mellitus. Neuro Endocrino Lett 36: 101-105, 2015.

3. Aires MB and Dos Santos AC: Effects of maternal diabetes on trophoblast cells. World J Diabetes 6: 338-344, 2015.

4. Sibley CP, Brownbill P, Glazier JD and Greenwood SL: Knowledge needed about the exchange physiology of the placenta. Placenta 64 (Suppl 1): S9-S15, 2018.

5. Leach L, Lammiman MJ, Babawale MO, Hobson SA Bromilou B, Lovat S and Simmonds MJ: Molecular organization of tight and adherens junctions in the human placental vascular tree. Placenta 21: 547-557, 2000.

6. Leach L, Taylor A and Sciota F: Vascular dysfunction in the diabetic placenta: Causes and consequences. J Anat 215: 69-76, 2009.

7. Sobrevia L, Salsoso R, Fuenzalida B, Barros E, Toledo L, Silva L, Pizarro C, Subiabre M, Villalobos R, Araos J, et al: Insulin is a key modulator of fetoplacental endothelium metabolic disturbances in gestational diabetes mellitus. Front Physiol 7: 119, 2016.

8. Subiabre M, Silva L, Toledo F, Paublo M, López MA, Boric MP and Sobrevia L: Insulin therapy and its consequences for the mother, foetus, and newborn in gestational diabetes mellitus. Biochim Biophys Acta Mol Basis Dis 1864: 2949-2956, 2018

9. Baumuller S, Lehnen H, Schmitz J, Fimmers R and Müller AM The impact of insulin treatment on the expression of vascular endothelial cadherin and beta-catenin in human fetoplacental vessels. Pediatr Dev Pathol 18: 17-23, 2015.

10. Bartakova V, Kollarova R, Kuricova K, Sebekova K, Belobradkova J and Kankova K: Serum carboxymethyl-lysine, a dominant advanced glycation end product, is increased in women with gestational diabetes mellitus. Biomed Pap Med Fac Univ Palacky Olomouc Czech Repub 160: 70-75, 2016.

11. Harsem NK, Braekke K, Torjussen T, Hanssen K and Staff AC: Advanced glycation end products in pregnancies complicated with diabetes mellitus or preeclampsia. Hypertens Pregnancy 27: 374-386, 2008
12. Guosheng L, Hongmei S, Chuan N, Haiying L, Xiaopeng Z and Xianqiong L: The relationship of serum AGE levels in diabetic mothers with adverse fetal outcome. J Perinatol 29: 483-488, 2009.

13. Stitt AW, Bhaduri T, McMullen CB, Gardiner TA and Archer DB: Advanced glycation end products induce blood-retinal barrier dysfunction in normoglycemic rats. Mol Cell Biol Res Commun 3: 380-388, 2000.

14. Guo XH, Huang QB, Chen B, Wang SY, Li Q, Zhu YJ, Hou FF, Fu N, Brunk UT and Zhao M: Advanced glycation end products induce actin rearrangement and subsequent hyperpermeability of endothelial cells. APMIS 114: 874-883, 2006.

15. Xie J, Mendez JD, Méndez-Valenzuela V and Aguilar-Hernández MM: Cellular signalling of the receptor for advanced glycation end products (RAGE). Cell Signal 25: 2185-2197, 2013.

16. Boulanger E, Grossin N, Wautier MP, Taamma R and Wautier JL: Mesothelial RAGE activation by AGEs enhances VEGF release and potentiates capillary tube formation. Kidney Int 71: 126-133, 2007.

17. Rodger MA, Gris JC, de Vries JIP, Martinelli I, Rey É, Schleussne E, Middeldorp S, Kaaja R, Langlois NJ, Ramsay $\mathrm{T}$, et al: Low-molecular-weight heparin and recurrent placenta-mediated pregnancy complications: A meta-analysis of individual patient data from randomised controlled trials. Lancet 388: 2629-2641, 2016.

18. Wat JM, Hawrylyshyn K, Baczyk D, Greig IR and Kingdom JC: Effects of glycol-split low molecular weight heparin on placental, endothelial, and anti-inflammatory pathways relevant to preeclampsia. Biol Reprod 99: 1082-1090, 2018.

19. Gasowska K, Naumnik B, Klejna K and Myśliwiec M: The influence of unfractionated and low-molecular weight heparins on the properties of human umbilical vein endothelial cells (HUVEC). Folia Histochem Cytobiol 47: 17-23, 2009.

20. Kevane B, Egan K, Allen S, Maguire P, Neary E, Lennon Á and Ní Áinle FN: Endothelial barrier protective properties of low molecular weight heparin: A novel potential tool in the prevention of cancer metastasis. Res Pract Thromb Haemost 1: 23-32, 2017.

21. Radu M and Chernoff J: An in vivo assay to test blood vessel permeability. J Vis Exp 73: e50062, 2013.

22. Livak KJ and Schmittgen TD: Analysis of relative gene expression data using real-time quantitative PCR and the 2(-Delta Delta C(T)) method. Methods 25: 402-408, 2001.

23. Meng Q, Shao L, Luo X, Mu Y, Xu W, Gao C, Gao L, Liu J and Cui Y: Ultrastructure of placenta of gravidas with gestational diabetes mellitus. Obstet Gynecol Int 2015: 283124, 2015.

24. Hayward CE, Jones RL and Sibley CP: Mechanisms of transfer across the human placenta. Placenta Intrauterine Environment 12: 121-133, 2008

25. Babawale MO, Lovat S, Mayhew TM, Lammiman MJ, James DK and Leach L: Effects of gestational diabetes on junctional adhesion molecules in human term placental vasculature. Diabetologia 43: 1185-1196, 2000.

26. Zhang Y, Zhao HJ, Xia XR, Diao FY, Ma X, Wang J, Gao L, Liu J, Gao C, Cui YG and Liu JY: Hypoxia-induced and HIF1 a-VEGF-mediated tight junction dysfunction in choriocarcinoma cells: Implications for preeclampsia. Clin Chim Acta 489: 203-211, 2019.

27. Li S and Yang H: Relationship between advanced glycation end products and gestational diabetes mellitus. J Matern Fetal Neonatal Med 32: 2783-2789, 2019.

28. Holman RR, Paul SK, Bethel MA, Matthews DR and Neil HA: 10-year follow-up of intensive glucose control in type 2 diabetes. New Engl J Med 359: 1577-1589, 2008.

29. Antonetti DA, Lieth E, Barber AJ and Gardner TW: Molecular mechanisms of vascular permeability in diabetic retinopathy. Semin Ophthalmol 14: 240-248, 1999.

30. Singh R, Barden A, Mori T and Beilin L: Advanced glycation end-products: A review. Diabetologia 44: 129-146, 2001.

31. Chilelli NC, Burlina S and Lapolla A: AGEs, rather than hyperglycemia, are responsible for microvascular complications in diabetes: A 'glycoxidation-centric' point of view. Nutr Metab Cardiovasc Dis 23: 913-919, 2013.

32. Svensjö E, Cyrino F, Michoud E, Ruggiero D, Bouskela E and Wiernsperger N: Vascular permeability increase as induced by histamine or bradykinin is enhanced by advanced glycation endproducts (AGEs). J Diabetes Complications 13: 187-190, 1999.

33. Wan $\mathrm{W}, \mathrm{Chen} \mathrm{H}$ and $\mathrm{Li} \mathrm{Y}$ : The potential mechanisms of $\mathrm{A} \beta$-receptor for advanced glycation end-products interaction disrupting tight junctions of the blood-brain barrier in Alzheimer's disease. Int J Neurosci 124: 75-81, 2014. 
34. Schmidt AM, Yan SD, Yan SF and Stern DM: The biology of the receptor for advanced glycation end products and its ligands. Biochim Biophys Acta 1498: 99-111, 2000.

35. Kisseleva T, Song L, Vorontchikhina M, Feirt N, Kitajewski J and Schindler C: NF-kappaB regulation of endothelial cell function during LPS-induced toxemia and cancer. J Clin Invest 116: 2955-2963, 2006.

36. Vandenbroucke E, Mehta D, Minshall R and Malik AB: Regulation of endothelial junctional permeability. Ann N Y Acad Sci 1123: 134-145, 2008.

37. Leach L, Gray C, Staton S, Babawale MO, Gruchy A, Foster C, Mayhew TM and James DK: Vascular endothelial cadherin and beta-catenin in human fetoplacental vessels of pregnancies complicated by Type 1 diabetes: Associations with angiogenesis and perturbed barrier function. Diabetologia 47: 695-709, 2004.

38. Obert E, Strauss R, Brandon C, Grek C, Ghatnekar G, Gourdie R and Rohrer B: Targeting the tight junction protein, zonula occludens-1, with the connexin43 mimetic peptide, aCT1, reduces VEGF-dependent RPE pathophysiology. J Mol Med (Berl) 95: 535-552, 2017.

39. Krishnasamy S, Ravi V, Rajaraman B, Kumar Thulasingam S, Dhevasena CS, Pathak A, Swaminathan K, Sundaresan M, Ayyappa KA, Arunkumar G, et al: Role of $\mathrm{VEGF}_{165 \mathrm{~b}} / \mathrm{VEGF}_{\mathrm{TOTAL}}$ ratio in gestational diabetes mellitus. Gynecol Endocrinol 35 811-814, 2019.

40. Melincovici CS, Boșca AB, Şuşman S, Mărginean M, Mihu C, Istrate M, Moldovan IM, Roman AL and Mihu CM: Vascular endothelial growth factor (VEGF)-key factor in normal and pathological angiogenesis. Rom J Morphol Embryol 59: 455-467, 2018.

41. Yan Y, Ji Y, Su N, Mei X, Wang Y, Du S, Zhu W, Zhang C, $\mathrm{Lu} Y$ and Xing XH: Non-anticoagulant effects of low molecular weight heparins in inflammatory disorders: A review. Carbohydr Polym 160: 71-81, 2017.
42. Abdel-Salam OM, Baiuomy AR, Ameen A and Hassan NS: A study of unfractionated and low molecular weight heparins in a model of cholestatic liver injury in the rat. Pharmacol Res 51: 59-67, 2005.

43. Nagy Z, Turcsik V and Blaskó G: The effect of LMWH (Nadroparin) on tumor progression. Pathol Oncol Res 15: 689-692, 2009.

44. Yalniz M, Demirel U, Orhan C, Bahcecioglu IH, Ozercan IH, Aygun C, Tuzcu M and Sahin K: Nadroparin sodium activates Nrf2/HO-1 pathway in acetic acid-induced colitis in rats. Inflammation 35: 1213-1221, 2012.

45. Delézay O, Hé Z, Sabido O, Hodin S, Bin V, Saleem MA, Mismetti $P$ and Delavenne X: Effects of heparin and derivatives on podocytes: An in vitro functional and morphological evaluation. J Cell Physiol: Jan 26, 2019 doi: 10.1002/jcp.28191 (Epub ahead of print).

46. Bentzer P, Fisher J, Kong HJ, Mörgelin M, Boyd JH, Walley KR, Russell JA and Linder A: Heparin-binding protein is important for vascular leak in sepsis. Intensive Care Med Exp 4: 33, 2016.

47. Takeuchi A, Yamamoto Y, Munesue S, Harashima A, Watanabe T, Yonekura $\mathrm{H}$, Yamamoto $\mathrm{H}$ and Tsuchiya $\mathrm{H}$ : Low molecular weight heparin suppresses receptor for advanced glycation end products-mediated expression of malignant phenotype in human fibrosarcoma cells. Cancer Sci 104: 740-749, 2013.

This work is licensed under a Creative Commons Attribution-NonCommercial-NoDerivatives 4.0 International (CC BY-NC-ND 4.0) License. 